\title{
Operation Time Variability in Multi Product Unpaced Production Lines
}

\author{
Suffi Ur Rehman and Lianyu Zheng
}

\begin{abstract}
In this paper a simulation study has been carried out on unpaced reliable and unreliable production lines having multiple products processing in batches with setup / changeover time. The aim of this study is to evaluate the design of multi product production lines operating under operation time variability for different products and compare the performance measures between reliable and unreliable lines. Simulation is performed on various line lengths having different mean buffer capacities in order to evaluate and observe the consistency of the results with the increase in number of stations and buffer capacities. Results obtained in this study have been analyzed in comparison with balanced lines of same configuration to find out the unbalanced scenarios / combinations caused by operation time variability in which unbalanced lines either outperforms or produce results closer to that of balanced lines.
\end{abstract}

Index Terms-Buffers, multiple products, operation time variability, plant simulation, unpaced production line.

\section{INTRODUCTION}

Due to competitive environment, major concern for most of the companies has been to get most out of their production system. Every company knows that efficiency improvements in the production line can produce considerable benefits. However, proper designing of production lines make a significant impact on its efficiency keeping in view the buffer allocation, number of stations, placement of workers etc. Most of the companies find it difficult to quickly implement flexible and multifaceted production lines that are capable of manufacturing a variety of products. Due to lots of unavoidable uncertainties at shop floor level, it becomes impossible to have a perfectly balanced line especially in multi product lines in which more than one product flows in the system in the form of batches requiring change of set up due to change of products In this type of scenarios, placement of workers creating bottleneck becomes even more important because operation time variability or coefficient of variation (CV) of one person vary with the change of product $\&$ change of setup.

Disruptions in the system may be caused by machine breakdown or failure which results temporary stoppage of the entire system. Breakdowns of single workstations or the entire line are particularly important in designing production lines because with the increase in number of stations in the line, the probability of all stations being operational at the same time decreases. These random failures result high idle time in the system due to starvation and blockage effect at

Manuscript received May 30, 2014; revised August 28, 2014.

Suffi Ur Rehman and Lianyu Zheng are with the Department of Industrial \& Manufacturing System Engineering, Beihang University (BUAA, Beijing University of Aeronautics \& Astronautics), Beijing, China (e-mail: suffirehman@hotmail.com; lyzheng@buaa.edu.cn). certain stations. When a failure occurs, a machine may not process any material, so the buffer upstream cannot lose material and the buffer downstream cannot gain material. An important consideration in the machine failure is up and down-time i.e reliability Mean Time Between Failure (MTBF) or Mean Time To Failure (MTTF) and maintainability Mean Time To Repair (MTTR), through which the availability or efficiency can be determined [1].

In order to ascertain the unbalancing factors in multi product lines and its effects on performance measures, this simulation study has been carried out on both reliable and unreliable lines. Comparison of performance measures between reliable and unreliable lines will emphasize the effects caused by MTTR and MTBF and will also help in distinguishing the effects caused by different imbalancing strategies. As mentioned above, in reality the unbalancing factors becomes unavoidable, the main purpose of this simulation study is to find out the possible scenarios for placement of workers which could either outperforms the results of balanced lines or produce results closer to that of balanced lines.

\section{LITERATURE REVIEW}

There has been substantial/considerable research conducted on the design of unpaced production lines keeping in view the buffer allocation, operating behavior, bottleneck sources and variability of mean processing time. Most the research in this area either comes under the development of algorithms/heuristics or general design rules. "Reference [2] considered the effects of unbalancing the line in terms of operation time variability and buffer capacities as a single source of unbalance. He concluded that in order to maximize output, middle stations must have lower variability as compared to the station at the start and end of the line". "Reference [3], [4] studied the effects of coefficient of variation of operation times on the allocation of storage space and also proposed a heuristic for optimal allocation of buffers in longer lines". "Reference [5] analyzed longer lines having single and multiple bottleneck stations, with lognormal service time distribution and several mean processing time and operation variability bottleneck location configurations while employing algorithm and simulation. They concluded that with the increase of bottleneck severity, more buffer units are attracted toward it. They further concluded that if both mean time and variance bottlenecks are located at the same position in the line, the bottleneck will have a stronger impact on buffer allocation". "Reference [6] considered the effects of unbalancing unreliable lines in terms of coefficient of variation and concluded different possible scenarios in which unbalanced lines perform either better or 
close to that of balanced lines".

"Reference [7]-[9] investigated the performance of unbalanced lines as regards to varying operational time variability while applying different optimization methods and concluded different possible scenarios in which unbalanced lines performs better than balanced lines. These algorithms have been applied to cases of lines having unequal operational time variability under a variety of operating conditions and for differing CV patterns". "Reference [10], [11] investigated the decline in line performance with the increase in process time variance having restricted buffer capacities". "Reference [12] investigated the consequence of single stations having higher variance at various positions in a line". "Reference [13] investigated three station unpaced production lines operating under varying buffer capacities and operation time variability together. They concluded that benefits can be achieved in the line by allocating greater interstation capacity around the more variable station". "Reference [14] compared the performance of single and double non mechanical flow lines operating under varying operation time variability $(\mathrm{CV}$ of $0.1,0.2 \& 0.3)$ and inter-station mean buffer capacities in different line lengths upto 30 stations. They concluded that double lines exhibited higher output as compared to equivalent single lines. "Reference [15] investigated the optimal allocation of buffer capacities in different line lengths operating under variable processing times and found that unlike bowl phenomenon for work allocations no single pattern for buffer allocations is optimal under all conditions and patterns for optimality of buffer allocation varies depending upon the extra storage space available. "Reference [16] investigated asynchronous flexible assembly systems (AFASs) using hybrid procedures having both analytical techniques and optimization methods and further elaborated it with some examples". "Reference [17] investigated the serial production lines operating under unreliable machines using exponential, Erlang and Rayleigh distributions with the aim of determining the smallest level of buffering required for the desired production rate".

However, in the past research on this topic considers only one type of product with no changeover / set up time. In this paper, imbalance of operation time variability in different scenarios has been employed on unpaced reliable and unreliable production lines having two products with changeover / set up time. Moreover, instead of having only one performance measure, this paper deals with the investigation of variations on multiple performance measures. The main objective of this study is to address the following questions:

- Effects of imbalance of operation time variability on the performance measures in unpaced multiple products reliable and unreliable lines

- Variation of performance measures against different line lengths and different inter-station buffer capacities

- Comparison of performance measures as regards to imbalance scenarios between reliable and unreliable lines

\section{Methodology}

In the subject simulation study, two products P1 \& P2 with different mean processing times and batch quantities have been processed in line lengths $(\mathrm{N})$ of four, seven, ten and fifteen number of stations with mean buffer (MB) capacities of two, three five and seven units. Lognormal distribution is applied at each station on processing time and set up time due to its positive skewness property and usage of the same distribution previous researchers on this subject. Detailed parameters are described in Table I.

\begin{tabular}{|c|c|c|c|c|c|}
\hline Products & $\begin{array}{c}\text { Batch } \\
\text { Size }\end{array}$ & $\begin{array}{c}\text { Mean } \\
\text { Processing } \\
\text { time } \\
\text { (mins) }\end{array}$ & $\begin{array}{c}\text { Set Up } \\
\text { Time } \\
\text { (mins) }\end{array}$ & $\begin{array}{c}\text { Line } \\
\text { Lengths }\end{array}$ & $\begin{array}{c}\text { Mean } \\
\text { Buffer } \\
\text { Capacity }\end{array}$ \\
\hline$P_{1}$ & 120 & 10 & 25 & $\begin{array}{c}4,7,10 \& \\
15\end{array}$ & $\begin{array}{c}2,3,5 \text { and } \\
7 \text { units }\end{array}$ \\
\hline$P_{2}$ & 40 & 30 & 25 & Stations & \\
\hline
\end{tabular}

Three main Performance measures/parameters of the line studied are as follows:

- Idle Time (IT): Percentage of time in which the worker or the station remain empty/idle due to failure, starvation or blockage caused by other stations. Average IT of the whole line is calculated at the end of simulation time.

- Production Rate (PR): The average output of a production process (machine, workstation, line, plant) per unit time is defined as the system's throughput, or production rate. For a plant, line, or workstation, production rate is the average quantity of goods produced per unit time [18].

- Work In Process (WIP): Work In Process inventory i.e the inventory which is in the production process (between source and drain) and has not yet been completed and transferred to the finished goods. In this paper total average WIP for the whole line is calculated at the end of the every simulation cycle.

\section{A. Simulation Parameters}

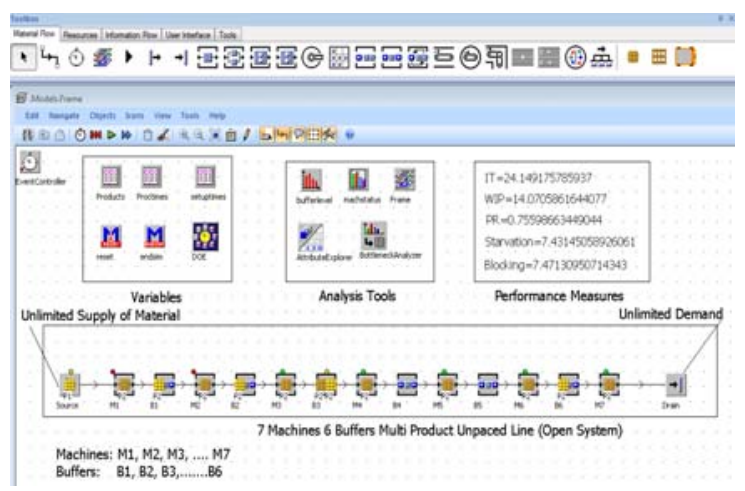

Fig. 1. Simulation model for 7 machines.

Siemens plant simulation software is used for this simulation study and programming has been done in simtalk language. Simulation model for line length seven stations having six intermediate buffers is shown in Fig. 1, Variations of different imbalancing scenarios on performance measures against multiple line lengths have been evaluated not only to observe the consistency of the results but also to investigate the design of production lines with the increase of number of stations. As proposed by [19], single output parameter (Throughput per hour) was selected for the preliminary simulation run in order to calculate the warm up period and line length with fifteen stations was 
selected in order to address the steady state condition for line lengths less than fifteen stations. Keeping in view the multi products scenario with change over time and random failure of the machines, simulation was run for longer period of 4560 hours units including extended warm up period of 1200 hours. However data after the initial warm up period is discarded and simulation was performed for the remaining time in order to get the results at steady state condition. Warm up period calculation is shown in Fig. 2. Data was collected at $95 \%$ confidence interval with three observations per run ( 3 replications) and the results averaged over three replicates.

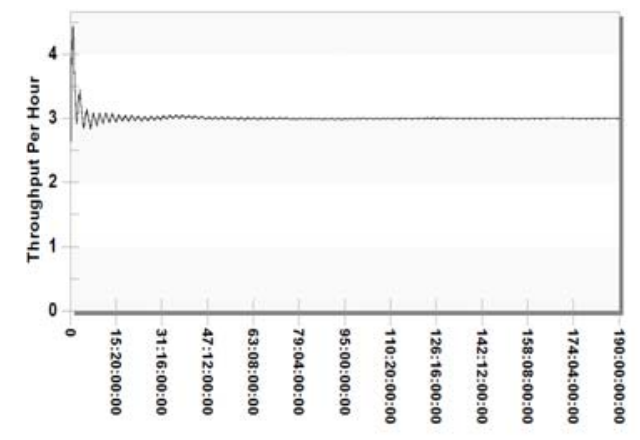

Fig. 2. Warm-up period.

\section{B. Model Assumptions}

Following assumptions have been made while simulating the unpaced multi product production lines:

- Stations are subject to starving and blocking, but the first station is never starved (Unlimited supply of raw materials) and the last station is never blocked (Unlimited demand) being the open production lines.

- Both the products are using the same buffers and there is no dedicated buffer for each product. Time to move the parts in and out of the storage buffers is negligible.

- The system consists of M stations arranged serially. M- 1 buffers separate each consecutive pair of stations

- Parts leave station 1, move to station 2, and so on sequentially through the line without any backtracking, bypassing, or re-entry.

As regards to unreliable lines, stations are subject to random mechanical failure \& repair. Time between failures and repair were both assumed to be exponentially distributed as concluded by [20] during the study of actual manufacturing systems. For this investigation mean time between failures (MTBF) and mean time to repair (MTTR) were 100 minutes and 10 minutes respectively, rates used by [6], [7] resulting in line efficiency or availability to be $91 \%$. All downtimes were considered to be operation dependent rather than simulation time i.e failure could only occur during the processing of job.

\section{Operation Time Variability}

Operation time variability with three level of coefficient of variation $(\mathrm{CV})$ in different scenarios including ascending $(/)$, descending $(\backslash)$, bowl $(\mathrm{V})$, inverted bowl $(\wedge)$ and zigzag $(\mathrm{W})$ were simulated while keeping the mean processing time constant for each product. Details of these scenarios for line length $4,7 \& 10$ stations are described in Table II.
- Ascending: Placement of the slow or bottleneck station at the end of line

- Descending: Placement of the slow or bottleneck station at the beginning of line

- Bowl: Placement of the slow or bottleneck stations at the beginning and end of line while placement of fast stations in the middle of the line.

- Inverted bowl: Placement of the slow stations in the middle of line

- Zigzag: Slow - Medium - Fast

It is pertinent to mention that more than 100 configurations have been simulated keeping in view the 25 configurations for both products against one mean buffer capacity resulting in total of more than 400 configurations for all line lengths.

TABLE II: SCENARIOS FOR CV IMBALANCE

\begin{tabular}{|c|c|c|c|}
\hline$N$ & 4 & 7 & 10 \\
\hline$/$ & $L_{1} L_{2} L_{2} L_{3}$ & $L_{1} L_{1} L_{2} L_{2} L_{2} L_{3} L_{3}$ & $L_{1} L_{1} L_{1} L_{2} L_{2} L_{2} L_{2} L_{3} L_{3} L_{3}$ \\
\hline$\backslash$ & $L_{3} L_{2} L_{2} L_{1}$ & $L_{3} L_{3} L_{3} L_{2} L_{2} L_{2} L_{1}$ & $L_{3} L_{3} L_{3} L_{2} L_{2} L_{2} L_{2} L_{1} L_{1} L_{1}$ \\
\hline$V$ & $L_{3} L_{1} L_{1} L_{2}$ & $L_{3} L_{3} L_{3} L_{1} L_{1} L_{1} L_{2}$ & $L_{3} L_{3} L_{3} L_{1} L_{1} L_{1} L_{1} L_{2} L_{2} L_{2}$ \\
\hline$\wedge$ & $L_{1} L_{3} L_{3} L_{2}$ & $L_{1} L_{1} L_{3} L_{3} L_{3} L_{2} L_{2}$ & $L_{1} L_{1} L_{1} L_{3} L_{3} L_{3} L_{3} L_{2} L_{2} L_{2}$ \\
\hline $\mathrm{W}$ & $L_{3} L_{2} L_{1} L_{3}$ & $L_{3} L_{2} L_{1} L_{3} L_{3} L_{1} L_{3}$ & $L_{3} L_{2} L_{1} L_{3} L_{2} L_{1} L_{3} L_{2} L_{1} L_{3}$ \\
\hline
\end{tabular}

$L_{1}$ : Level 1 with CV of 0.1

$L_{2}$ : Level 2 with CV of 0.3

$L_{3}$ : Level 3 with $\mathrm{CV}$ of 0.5

\section{RESUlTS AND DisCUSSION}

Results obtained in this simulation study in terms of Idle Time, Production Rate and Work In process inventory for reliable and unreliable lines are presented in subsequent sections. It is pertinent to mention that some of the key combinations having diverse effects on the performance measures have been presented and discussed. However complete data can be made available upon requirement.

\section{A. Idle Time}

Results of idle time for reliable and unreliable lines are presented in Table III and Table IV respectively. Following important findings/observations can be made from the results:-

TABLE III: IDLE TIME FOR RELIABLE LINES

\begin{tabular}{|c|c|c|c|c|c|c|c|}
\hline \multirow{2}{*}{$\mathrm{CV}$} & $P_{1}$ & V & 1 & $V$ & $Z$ & $\Lambda$ & 0.3 \\
\hline & $P_{2}$ & V & $V$ & 1 & $\wedge$ & $\wedge$ & 0.3 \\
\hline $\mathrm{N}$ & $\mathrm{MB}$ & & & & & & \\
\hline \multirow{4}{*}{4} & 2 & 7.950 & 8.744 & 8.767 & 10.654 & 12.036 & 8.63 \\
\hline & 3 & 6.387 & 7.001 & 7.117 & 9.883 & 8.833 & 7.08 \\
\hline & 5 & 4.477 & 5.053 & 5.173 & 6.620 & 7.536 & 5.10 \\
\hline & 7 & 3.407 & 3.869 & 4.082 & 5.170 & 6.026 & 3.84 \\
\hline \multirow{4}{*}{7} & 2 & 12.892 & 13.368 & 13.325 & 15.368 & 16.108 & 12.37 \\
\hline & 3 & 10.280 & 10.722 & 10.732 & 12.831 & 13.366 & 10.15 \\
\hline & 5 & 7.147 & 7.519 & 7.758 & 9.179 & 9.826 & 6.67 \\
\hline & 7 & 5.670 & 5.882 & 6.183 & 6.835 & 7.610 & 4.72 \\
\hline \multirow{4}{*}{10} & 2 & 16.224 & 16.471 & 16.321 & 18.343 & 18.959 & 15.38 \\
\hline & 3 & 12.846 & 13.210 & 13.099 & 15.132 & 15.479 & 12.43 \\
\hline & 5 & 8.969 & 9.191 & 9.443 & 10.242 & 10.972 & 7.47 \\
\hline & 7 & 7.165 & 7.300 & 7.602 & 7.437 & 8.423 & 5.09 \\
\hline \multirow{4}{*}{15} & 2 & 20.558 & 20.598 & 20.039 & 22.028 & 22.237 & 19.48 \\
\hline & 3 & 16.255 & 16.433 & 16.081 & 17.892 & 17.931 & 15.23 \\
\hline & 5 & 11.422 & 11.500 & 11.802 & 11.465 & 12.092 & 8.25 \\
\hline & 7 & 9.067 & 9.087 & 9.266 & 9.044 & 9.159 & 5.41 \\
\hline
\end{tabular}


TABLE IV: IDLE TIME FOR UNRELIABLE LINES

\begin{tabular}{|c|c|c|c|c|c|c|c|}
\hline \multirow{2}{*}{$\mathrm{CV}$} & $P_{1}$ & $\bar{V}$ & 1 & $\bar{V}$ & $\bar{Z}$ & $\Lambda$ & 0.3 \\
\hline & $P_{2}$ & $\bar{V}$ & $\bar{V}$ & 1 & $\Lambda$ & $\Lambda$ & 0.3 \\
\hline $\bar{N}$ & $\overline{\mathrm{MB}}$ & & & & & & \\
\hline \multirow{4}{*}{4} & 2 & 20.845 & 1.391 & 21.435 & 22.952 & 24.023 & 21.40 \\
\hline & 3 & 18.797 & 19.056 & 19.124 & 20.390 & 21.070 & 19.02 \\
\hline & 5 & 16.345 & 16.512 & 16.754 & 17.737 & 18.375 & 16.56 \\
\hline & 7 & 14.692 & 15.041 & 15.156 & 16.046 & 16.609 & 15.03 \\
\hline \multirow{4}{*}{7} & 2 & 25.791 & 26.074 & 26.154 & 27.542 & 28.130 & 25.67 \\
\hline & 3 & 23.056 & 23.286 & 23.378 & 24.383 & 24.927 & 22.75 \\
\hline & 5 & 19.172 & 19.447 & 19.639 & 20.835 & 21.049 & 19.11 \\
\hline & 7 & 6.872 & 17.084 & 17.188 & 18.101 & 18.507 & 16.64 \\
\hline \multirow{4}{*}{10} & 2 & 29.164 & 29.359 & 29.277 & 30.575 & 31.021 & 28.71 \\
\hline & 3 & 25.585 & 25.692 & 25.764 & 27.086 & 27.286 & 25.47 \\
\hline & 5 & 21.022 & 21.077 & 21.276 & 22.357 & 22.645 & 20.82 \\
\hline & 7 & .173 & 18.281 & 18.706 & 19.230 & 19.868 & 17.35 \\
\hline \multirow{4}{*}{15} & 2 & .197 & 33.135 & 32.892 & 34.363 & 34.441 & 32.48 \\
\hline & 3 & 28.977 & 28.897 & 28.746 & 30.026 & 30.209 & 28.27 \\
\hline & 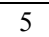 & 23.069 & 23.229 & 23.125 & 23.787 & 24.162 & 22.13 \\
\hline & 7 & 19.800 & 19.764 & 20.078 & 20.080 & 20.641 & 18.11 \\
\hline
\end{tabular}

- Idle time increases with the increase in number of stations and decreases with the increase of mean buffer capacities in both reliable and unreliable lines. However, comparison of reliable and unreliable lines indicates higher idle time in unreliable lines due to random failures.

- Bottleneck stations at the start and end of the line as regards to operation time variability for both the products produces good results in terms of less idle time in both reliable and unreliable lines. In shorter line lengths, this arrangement produces results which are either quite close or even better than balanced configuration. However, with the increase of number of stations and buffer capacities this effect diminishes although it still produces better results than the other imbalancing scenarios.

- Bottleneck stations present in the middle of the line as regards to operation time variability for both the products produces worst results in both reliable and unreliable setup.

- Analysis of the breakdown of idle time as regards to blocking and starvation effect for best, worst and balanced configuration indicates that placement of fast stations in the middle of the line reduce the blocking effect to some extent resulting in less idle time, however placement of the slowest stations in the middle of the line increase both the blocking and starvation effect resulting in high idle time. This effect is quite similar in both reliable and unreliable lines. Breakdown of idle time in terms of blocking and starvation for line length of four stations (unreliable lines) with mean buffer capacity of two units for bowl, inverted bowl and balanced configuration is mentioned below for more clarification of the above mentioned fact:-

$\begin{array}{lcc} & \text { Blocking }(\%) & \text { Starvation (\%) } \\ \text { Bow }(V-V) & 5.025 & 6.628 \\ \text { Inv. Bowl }(\wedge-\wedge) & 7.580 & 7.252 \\ \text { Balanced } & 6.032 & 6.182\end{array}$

- Generally speaking good results have been dominated in combinations involving bowl arrangement for any of the product combined with descending arrangement and worst results have been dominated in combinations involving inverted arrangement of any of the product combined with zigzag arrangement.
- Comparison of reliable and unreliable lines mean idle time against different scenarios as seen in Fig. 3 depicts that similar configurations produces best and worst results in both reliable and unreliable lines.

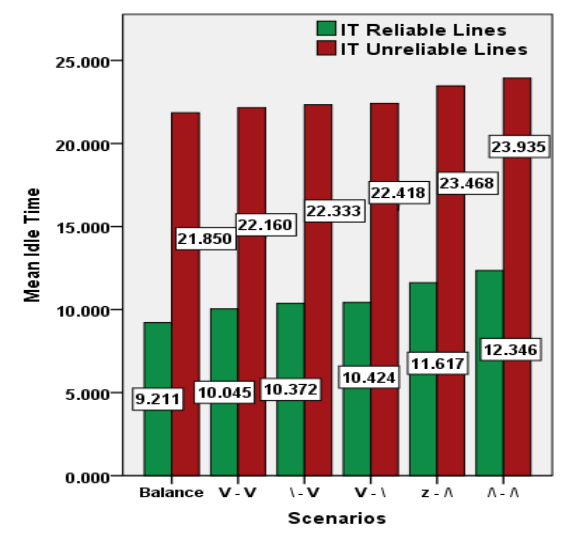

Fig. 3. Mean Idle Time against different scenarios.

\section{B. Production Rate}

Results of production rate for reliable and unreliable lines are presented in Table V and Table VI respectively. Similar scenarios produce better results for production rate as in case of idle time. Following important findings/observations can be made from the results:

TABLE V: PRODUCTION RATE FOR RELIABLE LINES

\begin{tabular}{|c|c|c|c|c|c|c|c|}
\hline \multirow{2}{*}{$\mathrm{CV}$} & $P_{1}$ & $V$ & 1 & $V$ & $Z$ & $\Lambda$ & 0.3 \\
\hline & $P_{2}$ & $V$ & $V$ & $\backslash$ & $\Lambda$ & $\wedge$ & 0.3 \\
\hline$N$ & $\mathrm{MB}$ & & & & & & \\
\hline \multirow{4}{*}{4} & 2 & 0.919 & 0.911 & 0.910 & 0.892 & 0.876 & 0.911 \\
\hline & 3 & 0.933 & 0.927 & 0.926 & 0.899 & 0.910 & 0.928 \\
\hline & 5 & 0.954 & 0.948 & 0.946 & 0.931 & 0.922 & 0.947 \\
\hline & 7 & 0.963 & 0.960 & 0.957 & 0.946 & 0.937 & 0.959 \\
\hline \multirow{4}{*}{7} & 2 & 0.868 & 0.863 & 0.864 & 0.846 & 0.839 & 0.874 \\
\hline & 3 & 0.894 & 0.892 & 0.893 & 0.869 & 0.866 & 0.897 \\
\hline & 5 & 0.927 & 0.923 & 0.920 & 0.906 & 0.902 & 0.931 \\
\hline & 7 & 0.941 & 0.938 & 0.935 & 0.931 & 0.923 & 0.951 \\
\hline \multirow{4}{*}{10} & 2 & 0.835 & 0.831 & 0.834 & 0.815 & 0.811 & 0.846 \\
\hline & 3 & 0.868 & 0.865 & 0.866 & 0.849 & 0.844 & 0.874 \\
\hline & 5 & 0.907 & 0.904 & 0.903 & 0.897 & 0.891 & 0.925 \\
\hline & 7 & 0.928 & 0.926 & 0.922 & 0.925 & 0.914 & 0.947 \\
\hline \multirow{4}{*}{15} & 2 & 0.795 & 0.794 & 0.797 & 0.778 & 0.777 & 0.802 \\
\hline & 3 & 0.835 & 0.833 & 0.838 & 0.823 & 0.822 & 0.847 \\
\hline & 5 & 0.884 & 0.882 & 0.879 & 0.883 & 0.876 & 0.915 \\
\hline & 7 & 0.906 & 0.906 & 0.905 & 0.907 & 0.906 & 0.944 \\
\hline
\end{tabular}

- Production Rate decreases with the increase in number of stations and increases with the increase in buffer capacities. However, production rate for unreliable lines decreases in comparison with reliable lines due to random failure rates.

- Bottleneck stations present at the start and end of the line (bowl configuration) as regards to operation time variability for both the products produce better results as compared to balanced configuration in both reliable and unreliable lines.

- Bottleneck or presence of high variable stations in the middle of the line for both the products produces worst results having less production rate.

- As explained in the analysis of idle time, placement of fast stations in the middle of the line tends to reduce the blocking effect at the start of the line resulting in increase of the production rate and placement of the slowest stations in the middle not only increase the 
blocking effect at the start of the line rather increase starvation for the end stations resulting in reduction in production rate in comparison with notionally balanced line.

- Generally speaking in both reliable and unreliable lines, bowl arrangement for any of the product combined with descending arrangement produces better results closer to that of balanced configuration and worst results have been dominated in combinations involving inverted arrangement of any of the product combined with zigzag arrangement.

- Similar configuration produces best and worst results in both reliable and unreliable lines in comparison with balanced lines as seen in Fig. 4.

TABLE VI: PRODUCTION RATE FOR UNRELIABLE LINES

\begin{tabular}{|c|c|c|c|c|c|c|c|}
\hline \multirow{2}{*}{$\mathrm{CV}$} & $P_{1}$ & $V$ & $\backslash$ & $V$ & $Z$ & $\wedge$ & 0.3 \\
\cline { 2 - 8 } & $P_{2}$ & $V$ & $V$ & $\backslash$ & $\wedge$ & $\wedge$ & 0.3 \\
\hline$N$ & $\mathrm{MB}$ & \multicolumn{7}{|c|}{} \\
\hline \multirow{4}{*}{4} & 2 & 0.791 & 0.785 & 0.785 & 0.767 & 0.758 & 0.786 \\
\cline { 2 - 8 } & 3 & 0.810 & 0.807 & 0.805 & 0.793 & 0.789 & 0.807 \\
\cline { 2 - 8 } & 5 & 0.834 & 0.832 & 0.829 & 0.822 & 0.814 & 0.831 \\
\cline { 2 - 8 } & 7 & 0.853 & 0.849 & 0.847 & 0.837 & 0.831 & 0.848 \\
\hline \multirow{4}{*}{7} & 2 & 0.739 & 0.737 & 0.735 & 0.725 & 0.719 & 0.741 \\
\cline { 2 - 8 } & 3 & 0.766 & 0.766 & 0.763 & 0.756 & 0.752 & 0.770 \\
\cline { 2 - 8 } & 5 & 0.805 & 0.802 & 0.801 & 0.792 & 0.789 & 0.806 \\
\cline { 2 - 8 } & 7 & 0.829 & 0.828 & 0.827 & 0.817 & 0.813 & 0.832 \\
\hline \multirow{4}{*}{10} & 2 & 0.706 & 0.704 & 0.705 & 0.694 & 0.689 & 0.713 \\
\cline { 2 - 8 } & 3 & 0.742 & 0.741 & 0.741 & 0.729 & 0.726 & 0.744 \\
\cline { 2 - 8 } & 5 & 0.789 & 0.788 & 0.786 & 0.774 & 0.771 & 0.791 \\
\cline { 2 - 8 } & 7 & 0.816 & 0.815 & 0.810 & 0.805 & 0.800 & 0.826 \\
\hline \multirow{4}{*}{15} & 2 & 0.666 & 0.666 & 0.669 & 0.656 & 0.655 & 0.674 \\
\cline { 2 - 8 } & 3 & 0.707 & 0.708 & 0.712 & 0.697 & 0.695 & 0.716 \\
\cline { 2 - 8 } & 5 & 0.767 & 0.765 & 0.769 & 0.761 & 0.760 & 0.778 \\
\hline & 7 & 0.800 & 0.800 & 0.799 & 0.798 & 0.793 & 0.817 \\
\hline
\end{tabular}

TABLE VII: WIP FOR RELIABLE LINES

\begin{tabular}{|c|c|c|c|c|c|c|c|}
\hline \multirow{2}{*}{$\mathrm{CV}$} & $P_{1}$ & $\backslash$ & $\backslash$ & $V$ & $/$ & $/$ & 0.3 \\
\cline { 2 - 8 } & $P_{2}$ & $\backslash$ & $Z$ & $V$ & $\wedge$ & $/$ & 0.3 \\
\hline$N$ & $\mathrm{MB}$ & \multicolumn{7}{|c|}{} \\
\hline \multirow{5}{*}{4} & 2 & 7.755 & 7.820 & 7.943 & 8.685 & 8.718 & 8.30 \\
\cline { 2 - 9 } & 3 & 9.305 & 9.340 & 9.620 & 10.454 & 10.386 & 9.92 \\
\cline { 2 - 9 } & 5 & 12.262 & 12.366 & 12.696 & 13.979 & 13.643 & 13.11 \\
\cline { 2 - 9 } & 7 & 14.578 & 15.011 & 15.093 & 17.357 & 16.882 & 15.81 \\
\hline \multirow{5}{*}{7} & 2 & 12.806 & 13.097 & 13.644 & 15.683 & 15.805 & 14.42 \\
\cline { 2 - 9 } & 3 & 15.946 & 16.450 & 17.312 & 19.267 & 19.195 & 17.84 \\
\cline { 2 - 9 } & 5 & 20.983 & 22.314 & 22.336 & 25.949 & 25.866 & 23.70 \\
\cline { 2 - 9 } & 7 & 24.372 & 26.618 & 25.567 & 31.953 & 32.320 & 28.47 \\
\hline \multirow{4}{*}{10} & 2 & 18.307 & 18.910 & 19.895 & 22.600 & 22.848 & 20.87 \\
\cline { 2 - 8 } & 3 & 23.087 & 24.321 & 25.164 & 27.879 & 28.019 & 25.95 \\
\cline { 2 - 8 } & 5 & 29.605 & 32.582 & 31.905 & 37.323 & 37.977 & 34.34 \\
\cline { 2 - 8 } & 7 & 33.809 & 37.893 & 35.721 & 45.462 & 47.554 & 40.98 \\
\hline \multirow{4}{*}{15} & 2 & 27.941 & 29.258 & 30.083 & 33.534 & 33.998 & 31.40 \\
\cline { 2 - 8 } & 3 & 34.657 & 37.501 & 37.612 & 41.276 & 41.847 & 39.23 \\
\cline { 2 - 8 } & 5 & 42.864 & 48.583 & 46.020 & 54.620 & 56.941 & 51.64 \\
\cline { 2 - 8 } & 7 & 48.743 & 55.519 & 51.259 & 66.405 & 71.829 & 60.94 \\
\hline
\end{tabular}

\section{Work in Process}

Results of WIP for reliable and unreliable lines are presented in table VII and table VIII respectively. Following important findings/observations can be made from the results:

- WIP increases with both the increase in number of stations and increase in buffer capacity in reliable and unreliable lines. It may be noted from the results that there is no significant difference between the values of
WIP for reliable and unreliable lines although it varies a little within different imbalance scenarios.

- Bottleneck stations present at the start of the line for both the products can be considered as best scenario in terms of less WIP.

- Bottleneck stations present at the end of line for both the products produces worst results having high WIP.

- Detailed analysis as regards to average buffer contents $(\mathrm{ABC})$ indicates that presence of slowest stations at the start of the line results in less utilization of buffer capacities at the start of the line in comparison with presence of bottleneck stations at the end of the line which results in higher average buffer contents at the start of the line resulting in high WIP. This effect is quite similar in both reliable and unreliable lines. Average buffer contents for first three buffers in line length seven stations (Unreliable lines) with mean buffer capacities of seven units are given below for better understating of the above mentioned facts.

$\begin{array}{cccc}B_{1} & B_{2} & B_{3} & \\ \text { Descending }(/-\backslash) & 3.986 & 3.682 & 3.313 \\ \text { Ascending }(/-/) & 4.568 & 4.388 & 4.084 \\ \text { Balanced } & 4.261 & 3.989 & 3.723\end{array}$

- Generally speaking configurations in which bottleneck stations are present at the start of the lines combined with either zigzag or bowl arrangement for any of the products produces better results and configurations having bottleneck stations at the end of the line combined with inverted bowl arrangement for any of the product results high WIP.

- Similar configuration produces best and worst results in both reliable and unreliable lines as seen in Fig. 5.

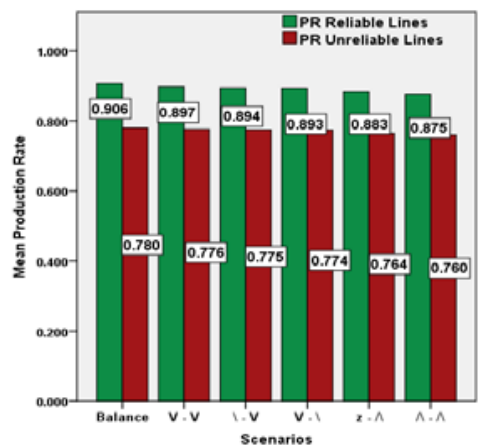

Fig. 4. Mean production rate against different scenarios.

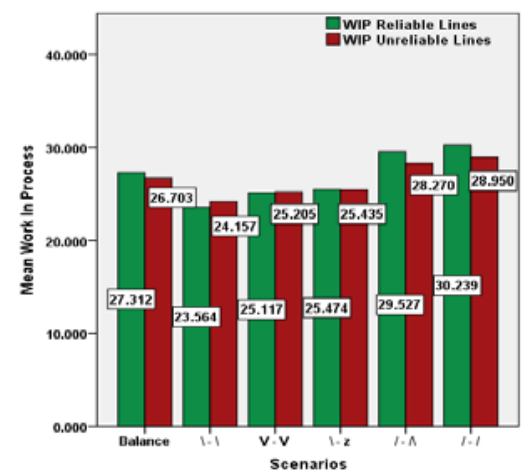

Fig. 5. Mean work in process against different scenarios.

\section{SUMMARY}

From the analysis of the above results, it can be observed 
that operation time variability imbalance for both the products do not have significant effect on the performance of the line. Effects of operational time variability is cushioned/absorbed by the even distribution of buffer capacities to some extent. However, few of the imbalancing scenarios deteriorate the line performance both in reliable and unreliable lines in comparison with the notionally balanced lines.

TABLE VIII: WIP FOR UNRELIABLE LINES

\begin{tabular}{|c|c|c|c|c|c|c|c|}
\hline \multirow{2}{*}{$\mathrm{CV}$} & $P_{1}$ & 1 & 1 & $V$ & 1 & 1 & 0.3 \\
\hline & $P_{2}$ & 1 & $Z$ & V & $\Lambda$ & 1 & 0.3 \\
\hline$N$ & MB & & & & & & \\
\hline \multirow{4}{*}{4} & 2 & 7.783 & 7.833 & 7.844 & 8.261 & 8.316 & 8.12 \\
\hline & 3 & 9.226 & 9.252 & 9.311 & 9.962 & 9.958 & 9.60 \\
\hline & 5 & 12.175 & 12.171 & 12.324 & 13.184 & 13.018 & 12.65 \\
\hline & 7 & 14.826 & 14.927 & 15.093 & 16.437 & 16.200 & 15.64 \\
\hline \multirow{4}{*}{7} & 2 & 12.962 & 13.177 & 13.335 & 14.733 & 14.880 & 13.98 \\
\hline & 3 & 15.956 & 16.273 & 16.498 & 18.165 & 18.199 & 17.08 \\
\hline & 5 & 21.626 & 22.102 & 22.147 & 24.547 & 24.675 & 23.22 \\
\hline & 7 & 26.118 & 27.145 & 26.791 & 30.716 & 30.970 & 28.38 \\
\hline \multirow{4}{*}{10} & 2 & 18.296 & 19.415 & 18.931 & 21.183 & 21.408 & 19.99 \\
\hline & 3 & 22.639 & 23.997 & 24.020 & 26.509 & 26.622 & 24.86 \\
\hline & 5 & 30.427 & 32.088 & 32.016 & 35.699 & 36.348 & 33.52 \\
\hline & 7 & 36.139 & 38.967 & 37.990 & 43.768 & 45.540 & 40.96 \\
\hline \multirow{4}{*}{15} & 2 & 27.341 & 28.716 & 28.626 & 31.882 & 32.309 & 30.25 \\
\hline & 3 & 34.314 & 36.048 & 36.287 & 39.560 & 40.410 & 37.73 \\
\hline & 5 & 44.778 & 48.851 & 47.468 & 53.233 & 55.483 & 50.54 \\
\hline & 7 & 51.900 & 57.377 & 54.622 & 64.487 & 68.869 & 60.73 \\
\hline
\end{tabular}

Bowl arrangement for both the products can be rated as best configuration as regards to idle time and production rate. However, for less WIP, descending arrangement for the products can be rated as best.

It can be clearly seen from the Fig. 6 and Fig. 7 that a single configuration in both reliable and unreliable lines cannot be rated as best for all the performance measure concurrently as descending arrangement for less WIP result higher idle time. However, as regards to less WIP bowl arrangement for both the products can be rated as second best which is the best configuration for improvement in Production Rate and Idle Time. So bowl arrangement for both the products can be chosen as a suitable arrangement in the given design parameters and amongst the few configurations simulated.

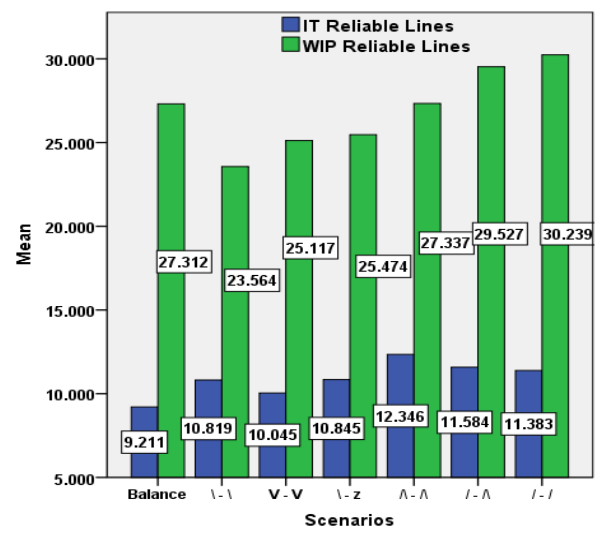

Fig. 6. Comparison of IT and WIP in reliable lines.

It is pertinent to mention that performance of the line is deteriorated with the increase in the number of stations having the same workload in all the imbalance scenarios simulated. Moreover, as regards to both idle time and production rate, bowl configuration effect for both the products also diminishes or lessens in longer line lengths.

Comparison of best and worst results in both reliable and unreliable lines clearly shows the importance of proper line design and placement of workers keeping in view the unavoidable uncertainties and competitive environment as even a very little improvement in the performance measures could result a large financial savings.

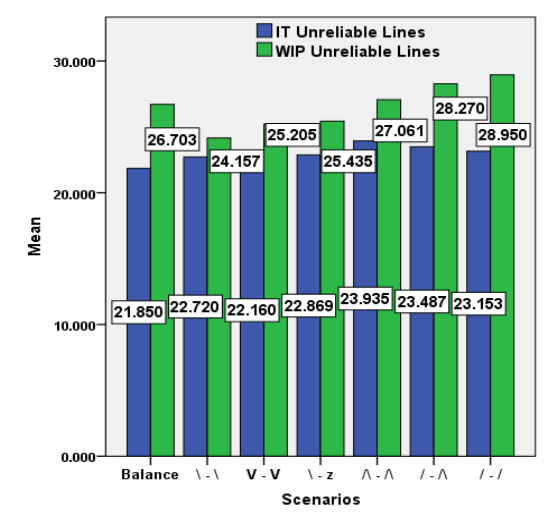

Fig. 7. Comparison of IT and WIP in unreliable lines.

\section{CONCLUSION}

A simulation modeling approach has been presented on multi product unpaced production lines involving changeover / set up time. The results demonstrate the effects on performance measures for the line operating with the same kind of arrangements of workers having different CV's for different products and also using the same buffers for multiple products. Although a limited number of configurations for operation time variability has been simulated but it still gives a general idea about the general behavior of such lines. However in these types of unpaced production lines, there are lots of other factors which are quite important e.g batch size, set up time, testing of components for the qualification of complete batch, shelf life of items etc. so it depends upon the company's policy how to operate keeping these scenarios in mind.

The main aim of this study was to analyze the behavior of multi product production lines operating under diverse unbalancing factors. There are some other parameter variations including set up time, mean processing time, buffer capacity variation etc. which can be employed from future research point of view.

\section{REFERENCES}

[1] G. A. Ronald and C. R. Standridge, Modeling and Analysis of Manufacturing Systems, John Wiley \& Sons Inc. 1993.

[2] T. E. E. Rayah, "The effect of inequality of interstage buffer capacities and operation time variability on the efficiency of production line systems," International Journal of Production Research, vol. 17, no. 1, pp. 77-89, 1979.

[3] F. S. Hillier and K. C. So, "The effect of the coefficient of variation of operation times on the allocation of storage space in production line systems," IIE Transactions, vol. 23, pp. 198-206, 1991.

[4] F. S. Hillier and K. C. So, "The effect of machine breakdowns and interstage storage on the performance of production line systems," International Journal of Production Research, vol. 29, pp. 2043-2055, 1991.

[5] I. Sabuncuoglu, E. Erel, and Y. Gocgun, "Analysis of serial production lines: characterization study and a new heuristic procedure 
for optimal buffer allocation," International Journal of Production Research, vol. 44, no. 13, pp. 2499-523, 2006.

[6] T. McNamara, S. Shaaban, and S. Hudson, "The effects of unbalancing operation time variability on the performance of unreliable lines," Journal of Manufacturing Technology Management, vol. 24 , no. 3, pp. 428-447, 2013.

[7] T. Altiok and S. Stidham, "The allocation of inter-stage buffer capacities in production lines," IIE Transactions, vol. 15, no. 4, pp. 292-299, 1983

[8] E. J. Muth, "The production rate of a series of work stations with variable service times," International Journal of Production Research, vol. 11, no. 12, pp. 155-169, 1973.

[9] G. Yamazaki, H. Sakasegawa, and J. G. Shanthikumar, "On optimal arrangement of stations in a tandem queuing system with blocking," Management Science, vol. 38, no. 1, pp. 137-53, 1992.

[10] A. D. Knott, "The inefficiency of a series of work stations: A simple formula," International Journal of Production Research, vol. 8, no. 2, pp. 109-119, 1970.

[11] S. Payne, N. Slack, and R. Wild, "A note on the operating characteristics of balanced and unbalanced production flow lines," International Journal of Production Research, vol. 9, no. 2, pp. 93-98, 1971.

[12] R. Kala and G. G. Hitchings, "The effect of performance time variance on a balanced four-station manual assembly line," International Journal of Production Research, vol. 11, no. 2, pp. 341, 1973.

[13] L. D. Smith and P. Brumbaugh, "Allocating Inter-station inventory capacity in unpaced production lines with heteroscedastic processing times," International Journal of Production Research, vol. 15, no. 2, pp. 163-172, 1977.

[14] R. Wild and N. D. Slack, "The operating characteristics of 'single' and 'double' non-mechanical flow line systems," International Journal of Production Research, vol. 11, no. 2, pp. 139-145,1973.

[15] F. S. Hillier, C. K. So, and R. Boling, "Notes: toward characterizing the optimal allocation of storage space in production line systems with variable processing times," Management Science, vol. 39, pp. 126-132, 1993.

[16] A. A. Bulgak and J. L. Sanders, "Modeling and design optimization of asynchronous flexible assembly systems with statistical process control and repair," International Journal of Flexible Manufacturing Systems, Kluwer Academic Publishers, vol. 3, no. 3/4, pp. 251-274, 1991.

[17] E. Enginarlar, J. Li, S. M. Meerkov, and R. Q. Zhang, "Buffer capacity for accommodating machine downtime in serial production lines," International Journal of Production Research, vol. 40, pp. 601-624, 2002
[18] W. J. Hopp and M. L. Spearman, Factory Physics, McGraw Hill, 2000.

[19] A. M. Law and W. D. Kelton, Simulation Modeling and Analysis, McGraw Hill, 2000

[20] R. R. Inman, "Empirical evaluation of exponential and independence assumptions in queuing models of manufacturing systems," Production and Operations Management, vol. 8, no. 4, pp. 409-32, 1999.

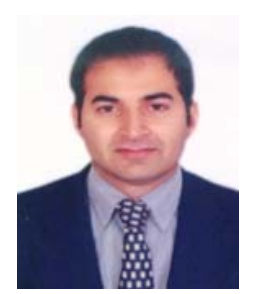

Suffi Ur Rehman was born in 1979 in Pakistan and received his B.S degree in mechanical engineering in 2001 from university of engineering and technology Lahore. $\mathrm{He}$ is currently doing master's in industrial engineering from Beihang University (BUAA, Beijing University of Aeronautics \& Astronautics) and having a background of professional experience of 10 years in industrial and production engineering. His research interests include system modeling \& simulation, manufacturing system management and material planning.

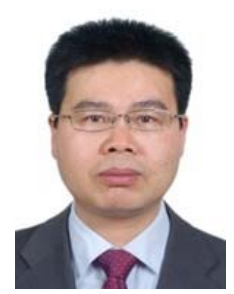

Lianyu Zheng was born in 1967, Jiangxi Province. He received the B.S., M.S. and Ph.D. degrees in mechanical engineering from Beihang University (BUAA, Beijing University of Aeronautics and Astronautics), Beijing, China, in 1989, 1993, 2001 respectively.

$\mathrm{He}$ has worked as a senior research associate (SRA), in the Department of Manufacturing Engineering and Engineering Management, City University of Hong Kong, China, from March to June in 2002. And as a visiting scholar, he has done research for one year (from October 2006 to September 2007) in the Department of Mechanical Engineering, University of Bath, UK. He is currently a professor and the head of the Department of Industrial and Manufacturing Systems Engineering, School of Mechanical Engineering and Automation, Beihang University.

Dr. Lianyu Zheng's current research interests include digital design and manufacturing (CAPP/CAM/CNC) and involved data standards (STEP, GPS-Geometrical Product Specification and Verification), dimension metrology and quality control, variation management based on key characteristics through product lifecycle, reconfigurable flexible manufacturing, and manufacturing systems modeling and simulation. $\mathrm{He}$ has published more than 70 papers including a number of international journal and conference papers, 3 edited books. 